\title{
Detection of Signal Discontinuities from Noisy Fourier Data
}

\author{
Adityavikram Viswanathan, Douglas Cochran \\ Department of Electrical Engineering \\ Arizona State University, Tempe, AZ 85287-5706, USA
}

\author{
Anne Gelb, Dennis M. Cates \\ Department of Mathematics and Statistics \\ Arizona State University, Tempe, AZ 85287-1804, USA
}

\begin{abstract}
The concentration method for identifying the locations, magnitudes, and signs of jump discontinuities in analog signals from truncated Fourier series is well established in mathematical literature. Its performance in the presence of noise on the Fourier data has only recently started to receive attention, however. This paper examines the performance of the concentration method in the presence of noise from a detection-theoretic point of view. In particular, receiver operating characteristics for the elemental problem of detecting a unit step discontinuity are developed for this method. Additionally, the problem of optimally combining data obtained from multiple concentration factors is addressed.
\end{abstract}

\section{INTRODUCTION}

The ability to detect abrupt changes in a signal is of fundamental importance in many signal processing applications. Frequently, one collects sampled data from either a spatial or temporal signal and seeks information about abrupt changes in the frequency spectrum (e.g., the presence of spectral lines). Less often, sampled data are collected in the frequency domain and abrupt changes in the temporal or spatial signal are sought. This is the case in magnetic resonance imaging (MRI), for example, where frequency domain (" $k$-space") samples are collected and edges of the physical-space image are of interest.

Given a finite collection of Fourier coefficients of a function containing jump discontinuities, the associated Fourier partial sum is smooth and exhibits Gibbs oscillations. When the number of Fourier coefficients available is relatively small, the Gibbs phenomenon significantly distorts the signal in neighborhoods of its discontinuities and thereby presents some challenges in identifying points of discontinuity even in the absence of noise. The concentration method, [1], [2], is a linear, non-parametric approach for directly approximating the jump function associated with a piecewise smooth function from a truncated Fourier series. Direct approximation of the jump function from Fourier data allows for significant savings in computational cost when compared to a two step process of Fourier reconstruction followed by physical space jump function approximation. Convergence and other fundamental mathematical characteristics of the concentration method have been studied in the deterministic setting, but its performance in the presence of noise has only recently started to receive attention [3], [4]. This paper examines the method's performance in the presence of noise from a detection-theoretic perspective.

This work was supported in part by National Science Foundation grants CNS 0324957, DMS 0510813 and FRG 0652833.
The remainder of this paper is organized as follows. Section II synopsizes the concentration method, the role of concentration factors in the method, and the method's foundations in the theory of generalized conjugate partial Fourier sums. Section III describes a detector based on the concentration method and using Fourier data corrupted by additive Gaussian noise. Extensions of the basic detector by combining multiple concentration factors and by testing a neighborhood of the putative point of discontinuity rather than at a single point are also discussed in this Section. Section IV presents performance data and Section V comments on the results obtained to date and some avenues for further investigation.

\section{The Concentration Method}

Let $f$ be a $2 \pi$-periodic, piecewise smooth function on $[-\pi, \pi)$. If $f\left(x^{+}\right)$and $f\left(x^{-}\right)$are well-defined right and left hand limits respectively of $f$ at every point $x$, the corresponding jump function $[f]$ is defined by

$$
[f](x):=f\left(x^{+}\right)-f\left(x^{-}\right)
$$

We are interested in computing an approximation to $[f]$, given the Fourier series coefficients

$$
\hat{f}_{k}=\frac{1}{2 \pi} \int_{-\pi}^{\pi} f(x) e^{-i k x} d x, k \in[-N, N]
$$

We note that $[f]$ represents a local phenomenon, while $\hat{f}$ is a global measure. To understand how jump data may be extracted from $\hat{f}$, consider, without loss of generality, that $f$ contains a single jump at $x=\zeta$. Integrating (2) by parts, we can show that

$$
\hat{f}(k)=[f](\zeta) \frac{e^{-i k \zeta}}{2 \pi i k}+\mathcal{O}\left(\frac{1}{k^{2}}\right)
$$

We observe that the jump value and location are contained in $\hat{f}$; however, the additional $\mathcal{O}\left(\frac{1}{k^{2}}\right)$ term, which is a manifestation of the global nature of Fourier data, complicates recovery. The concentration method, [1], [2], computes an approximation to $[f]$ using a partial sum of the form

$$
S_{N}^{\sigma}[f](x)=i \sum_{k=-N}^{N} \hat{f}_{k} \operatorname{sgn}(k) \sigma\left(\frac{|k|}{N}\right) e^{i k x}
$$

This formulation is referred to as the generalized conjugate partial Fourier sum, with $\sigma_{k, N}(\eta)=\sigma\left(\frac{|k|}{N}\right)$ known as concentration factors. For example, substituting (3) in (4), and using 
$\sigma\left(\frac{|k|}{N}\right)=\frac{\pi|k|}{N}$, we can show that

$$
S_{N}^{\sigma}[f](x)=[f](\zeta) \frac{1}{2 N} \sum_{k=-N}^{N} e^{i k(x-\zeta)}+\mathcal{O}\left(\frac{\log N}{N}\right)
$$

The first term is a Dirichlet kernel, scaled by the jump value and localized at the jump location, while the second term results from the global nature of $\hat{f}$. The concentration method provides a family of concentration factors to minimize the contribution of this error term, while preserving the jump localization. These factors are required to satisfy certain conditions, [2], in order to be admissible. Under these conditions, we have the following (concentration property):

$$
S_{N}^{\sigma}[f](x)=[f](x)+\mathcal{O}(\epsilon)
$$

where $\epsilon=\epsilon(N)>0$ is small. Details of the convergence analysis can be found in [1] and [2]. Table I lists three popular concentration factors while Figure 1 shows the jump function approximation for a unit step function using each of the three factors.

TABLE I

POPULAR CONCENTRATION FACTORS

\begin{tabular}{|c|c|}
\hline Factor & Expression \\
\hline Trigonometric & $\sigma_{T}(\eta)=\frac{\pi \sin (\alpha \eta)}{\operatorname{Si}(\alpha)}$ \\
& $\operatorname{Si}(\alpha)=\int_{0}^{\alpha} \frac{\sin (x)}{x} d x$ \\
\hline Polynomial & $\begin{array}{c}\sigma_{P}(\eta)=-p \pi \eta^{p} \\
\text { is the order of the factor }\end{array}$ \\
\hline Exponential & $\begin{array}{c}\sigma_{E}(\eta)=C \eta \exp \left(\frac{1}{\alpha \eta(\eta-1)}\right) \\
C-\text { normalizing constant; } \alpha-\text { order } \\
\end{array}$ \\
& $C=\frac{\pi}{\int_{\frac{1}{N}}^{1-\frac{1}{N}} \exp \left(\frac{1}{\alpha \tau(\tau-1)}\right) d \tau}$ \\
\hline
\end{tabular}

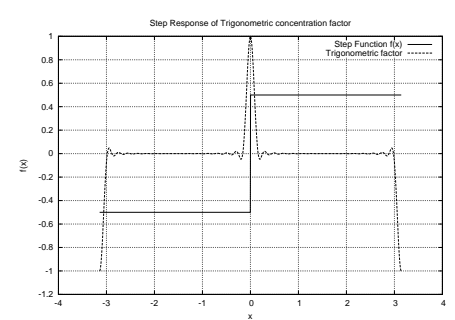

(a) Trigonometric factor

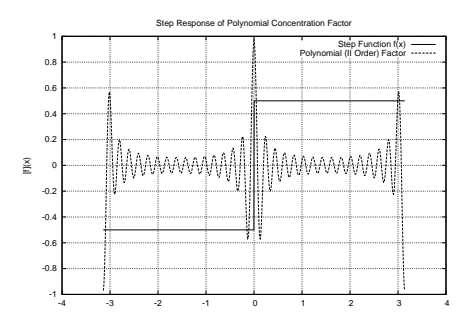

(b) Polynomial factor

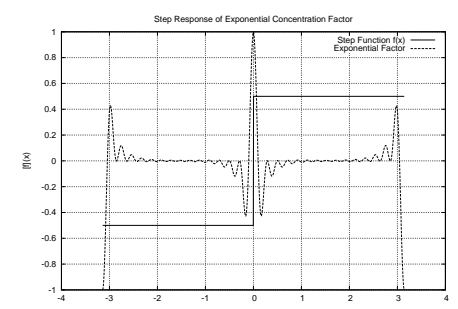

(c) Exponential factor

Fig. 1. Jump function approximations for a unit step function using different concentration factors $(N=32)$

\section{Detector Design}

This section presents the design of a detector that uses jump function approximation in conjunction with a matched filter to detect discontinuities in piecewise smooth functions. The detector operates on a finite set of Fourier coefficients $\hat{f}_{k}$, $k=-N, \ldots, N$, that are assumed to be corrupted by additive zero-mean white (complex) Gaussian noise $\hat{\mathbf{v}}_{k}$ with variance $\rho^{2}$. Hence, the observed coefficients are complex Gaussian random variables $\hat{\mathrm{g}}_{k}$ given by

$$
\hat{\mathbf{g}}_{k}=\hat{f}_{k}+\hat{\mathbf{v}}_{k} \quad k=-N, \ldots, N
$$

Linearity of the concentration method implies

$$
S_{N}^{\sigma}[\mathbf{g}]=S_{N}^{\sigma}[f]+S_{N}^{\sigma}[\mathbf{v}]
$$

so that the variate $S_{N}^{\sigma}[\mathbf{g}](x)$ is Gaussian with mean

$$
\mathrm{E}\left[S_{N}^{\sigma}[\mathbf{g}](x)\right]=S_{N}^{\sigma}[f](x)
$$

for each $x$. In particular, the noise component does not bias the jump function approximation.

The covariance of an approximation $S_{N}^{\sigma_{p}}[\mathbf{g}]\left(x_{a}\right)$ at a point $x_{a}$ using concentration factor $\sigma_{p}$ with an approximation $S_{N}^{\sigma_{q}}[\mathbf{g}]\left(x_{b}\right)$ at another point $x_{b}$ using concentration factor $\sigma_{q}$ is

$$
\left(C_{\mathbf{v}}\right)_{p, q}^{x_{a}, x_{b}}=\mathrm{E}\left[\left(S_{N}^{\sigma_{p}}[\mathbf{v}]\left(x_{a}\right)\right)\left(S_{N}^{\sigma_{q}}[\mathbf{v}]\left(x_{b}\right)\right)^{*}\right]
$$

Direct calculation yields

$$
\left(C_{\mathbf{v}}\right)_{p, q}^{x_{a}, x_{b}}=\rho^{2} \sum_{k=-N}^{N} \sigma_{p}\left(\frac{|k|}{N}\right) \sigma_{q}\left(\frac{|k|}{N}\right) e^{i k\left(x_{a}-x_{b}\right)}
$$

$\left(C_{\mathbf{v}}\right)_{p, q}^{x_{a}, x_{b}}$ is Hermitian symmetric, but not Toeplitz, due to the presence of the concentration factors $\sigma_{p}\left(\frac{|k|}{N}\right)$ and $\sigma_{q}\left(\frac{|k|}{N}\right)$.

\section{A. Formulation of the detection problem}

For the purpose of detector design, the unit step function with step at $x=0$ will be used as the elemental function and the objective of the detector will be to test at, or in a neighborhood of, $x=0$ to distinguish between its presence $\left(\mathcal{H}_{1}\right)$ and absence $\left(\mathcal{H}_{0}\right)$. Given $L>0$ pairs $\left(x_{i}, \sigma_{i}\right)$ of points $x_{i}$ and concentration factors $\sigma_{i}$, an $L$-vector $M$ of approximation values is formed as

$$
M=\left(S_{N}^{\sigma_{1}}[f]\left(x_{1}\right), \ldots, S_{N}^{\sigma_{L}}[f]\left(x_{L}\right)\right)^{T}
$$

Similarly, a random $L$-vector $\mathbf{Y}=$ $\left(S_{N}^{\sigma_{1}}[\mathbf{g}]\left(x_{1}\right), \ldots,\left(S_{N}^{\sigma_{L}}[\mathbf{g}]\left(x_{L}\right)\right)^{T}\right.$ represents the noisy test data. By virtue of (8), the covariance matrix $C_{\mathbf{Y}}$ of $\mathbf{Y}$ is exactly the covariance matrix $C_{\mathbf{v}}$ of the noise component $\mathbf{V}$ of $\mathbf{Y}$, which can be obtained from (10). Hence the detection problem on the data $\mathbf{Y}$ is

$$
\begin{aligned}
& \mathcal{H}_{0}: \mathbf{Y}=\mathbf{V} \quad \sim \mathcal{C N}\left[0, C_{\mathbf{v}}\right] \\
& \mathcal{H}_{1}: \mathbf{Y}=M+\mathbf{V} \sim \mathcal{C N}\left[M, C_{\mathbf{v}}\right]
\end{aligned}
$$

where $\mathcal{C N}[A, C]$ represents a multivariate (complex) Gaussian distribution with mean $A$ and covariance matrix $C$. To maximize the probability of detection $P_{\mathrm{d}}$ for a given false alarm 
rate $P_{\text {fa }}$, the Neyman-Pearson criterion, [5], yields a detector of the form

$$
M^{T} C_{\mathbf{v}}^{-1} \mathbf{Y}>\gamma
$$

where $\gamma$ is a threshold. This is a (generalized) matched filter whose performance is given by the relation

$$
P_{\mathrm{d}}=Q\left(Q^{-1}\left(P_{\mathrm{fa}}\right)-\sqrt{M^{T} C_{\mathbf{v}}^{-1} M}\right)
$$

with $Q(x):=\int_{x}^{\infty} \frac{1}{\sqrt{2 \pi}} \exp \left(-\frac{t^{2}}{2}\right) d t$ denoting the complementary cumulative distribution function. For a given false alarm rate, the quantity $M^{T} C_{\mathbf{v}}^{-1} M$ governs the performance of the detector and hence may be interpreted as a signal-tonoise ratio (SNR).

\section{B. Factors influencing detector performance}

The SNR $M^{T} C_{\mathbf{v}}^{-1} M$ is a function of the signal vector $M$ and the noise variance $\rho^{2}$. In turn, $M$ depends on the choice of pairs $\left(x_{i}, \sigma_{i}\right)$ of measurement points and concentration factors that define the measurement data. Results presented in Section IV show that it is indeed advantageous to use a combination of concentration factors in selecting the signal vector $M$. The intuition behind this is illustrated by Figure 1, where each response shows distinctive characteristics such as mainlobe width and oscillation pattern away from the jump point.

Tables II and III seek to illustrate this behavior. Table II shows three candidate signal vectors computed using the Trigonometric factor, and their corresponding values of $M^{T} C_{\mathbf{v}}^{-1} M$. Table III shows the effect of choice of concentration factors when location $\left\{x_{i}\right\}_{i=1}^{5}$ is fixed (Notation used: T - Trigonometric factor, P - Polynomial factor, E - Exponential factor). For the results shown in Table III, the set of $x_{i}$ chosen was $\{-0.193,-0.096,0,0.096,0.193\}$.

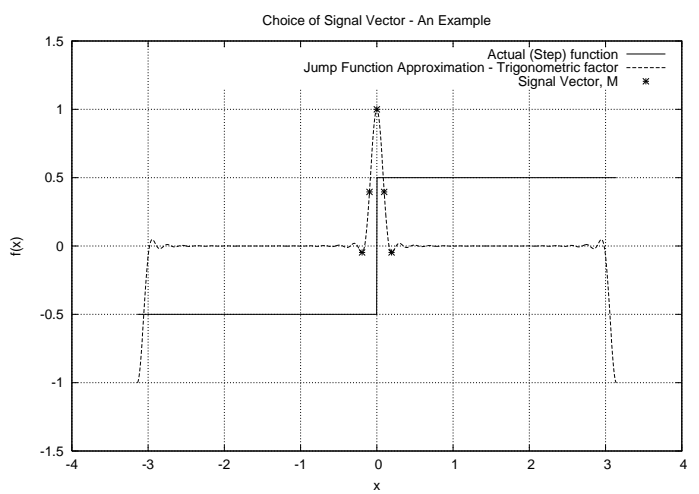

Fig. 2. Example of a signal vector $(\mathrm{N}=32$, Trigonometric factor $)$

\section{REsults}

Figure 3(a) shows receiver operating characteristic (ROC) curves for a detector designed as per Section III. The solid curve was obtained theoretically from (12); Monte Carlo simulations were used to obtain the empirical dashed curve. In both
TABLE II

CHOICE OF LOCATIONS $x_{i}$ AND EFFECT ON PERFORMANCE USING ONLY THE TRIGONOMETRIC CONCENTRATION FACTOR $\left(L=5, N=32, \rho^{2}=7.5\right)$

\begin{tabular}{|c|c|}
\hline Choice of $x_{i}$ & $\mathbf{M}^{\mathbf{T}} \mathbf{C}_{\mathbf{v}}^{-\mathbf{1}} \mathbf{M}(\mathrm{dB})$ \\
\hline$(-0.294,-0.196,0,0.196,0.294)$ & 13.498 \\
maxima/minima locations in $S_{N}^{\sigma}[f](x)$ & \\
\hline $\begin{array}{c}(-0.193,-0.096,0,0.096,0.193) \\
\text { grid points spaced } \frac{1}{2 N+1} \text { apart }\end{array}$ & 14.656 \\
\hline $\begin{array}{c}(-0.348,-0.173,0,0.173,0.348) \\
\text { optimal selection (numerically computed) }\end{array}$ & 16.396 \\
\hline
\end{tabular}

cases, the parameters used were $L=3, N=32, \rho^{2}=7.5$. The exponential concentration factor was used and the set of $x_{i}$ was chosen to be $\left\{\begin{array}{lll}-\delta & 0 & \delta\end{array}\right\}$ with $\delta=\frac{1}{2 N+1}$. The SNR for this selection of parameters is $9.0283 \mathrm{~dB}$. Close agreement between the simulated and theoretical results is observed. Figure 3(b) shows the ROC curve for the same detector at a noise variance $\rho^{2}=3.5$. The SNR for this case is $12.338 \mathrm{~dB}$.

Figure 4 illustrates how performance can be improved by using a combination of concentration factors rather than a single concentration factor. The common parameters for both the detectors are $L=3, N=32$, and $\rho^{2}=7.5$. The dashed curve corresponds to the detector using the exponential concentration factor chosen at points $\left(\begin{array}{lll}-\delta & 0 & \delta\end{array}\right)$ with $\delta=\frac{1}{2 N+1}$. The corresponding SNR is $9.0283 \mathrm{~dB}$. The solid curve corresponds to a detector using all three concentration factors with $x_{i}=0$. The SNR in this case is $13.434 \mathrm{~dB}$. Finally, Figure 5 shows the detection of jumps in a test signal with multiple jumps, and jumps of varying magnitude and sign. This particular example uses the trigonometric concentration factor with $x_{i}$ chosen to be $\left(\begin{array}{lll}-\delta & 0 & \delta\end{array}\right)$ and $\delta=\frac{1}{2 N+1}$. Additional processing in the form of accounting for negative valued jumps and computing the jump values have been performed here. Note the presence of false alarms near some of the correctly characterized points of discontinuity, suggesting the need for mitigation of sidelobe effects in non-idealized problems: the $\mathcal{H}_{0}$ assumption used above in characterizing detector performance is not satisfied when testing at points very near a point of discontinuity.

\section{DISCUSSION}

This paper has presented an approach for detecting discontinuities in a piecewise-smooth analog signal starting from a finite set of noisy Fourier coefficients. The approach is based on the concentration method, whose performance in the presence of noise has only recently started to receive attention. By simultaneously employing data from multiple

TABLE III

CHOICE OF CONCENTRATION FACTORS AND EFFECT ON PERFORMANCE USING A FIXED SET OF LOCATIONS $x_{i}\left(L=5, N=32, \rho^{2}=7.5\right)$

\begin{tabular}{|c|c|}
\hline $\begin{array}{c}\text { Choice of concentration factors } \\
\left(\sigma_{1} \sigma_{2} \sigma_{3} \sigma_{4} \sigma_{5}\right)\end{array}$ & $\mathbf{M}^{\mathbf{T}} \mathbf{C}_{\mathbf{v}}^{-\mathbf{I}} \mathbf{M}(\mathrm{dB})$ \\
\hline$($ T P E P T) & 11.285 \\
\hline$($ T P E T P) & 13.123 \\
\hline (T P P P T) & 14.727 \\
\hline
\end{tabular}




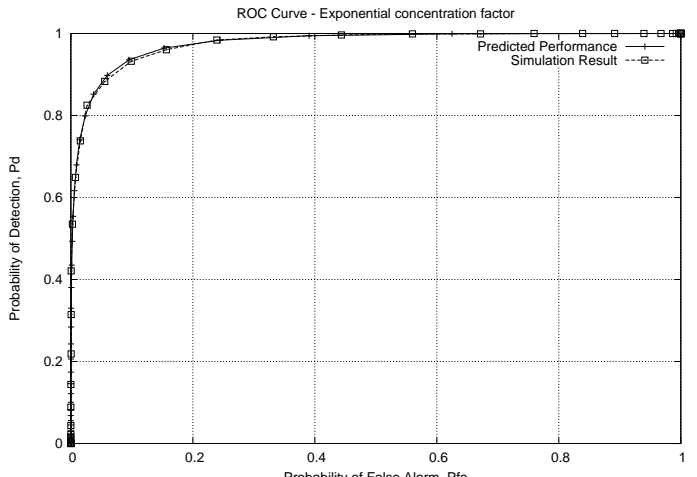

(a) $\rho^{2}=7.5$

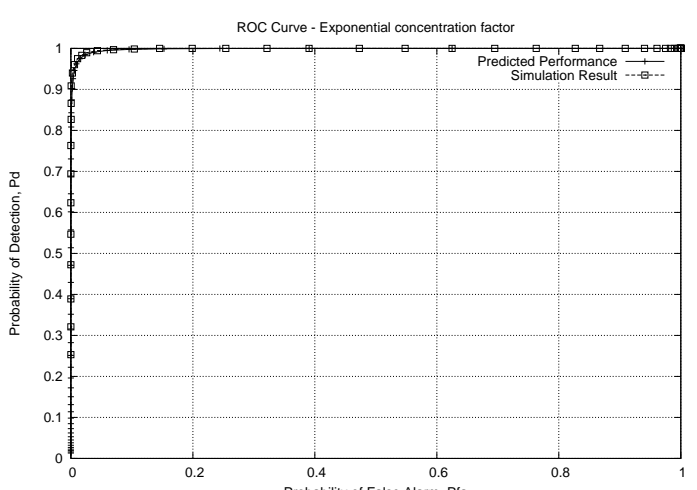

(b) $\rho^{2}=3.5$

Fig. 3. ROC curve ( $L=3, N=32$, Exponential factor)

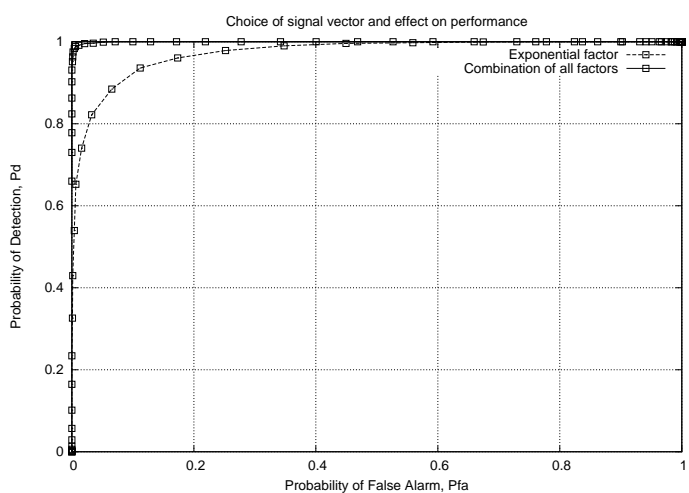

Fig. 4. Single concentration factor versus a combination of factors $\left(L=3, N=32, \rho^{2}=7.5\right)$

concentration factors applied at multiple points in a statistical hypothesis test, the approach described here capitalizes on the availability of a multiplicity of concentration factors that exhibit different behaviors near points of discontinuity.

The SNR introduced in Section III provides a metric for selection of sets of test points and concentration factors that will provide good performance. It should ultimately be possible to optimize this selection process, possibly all the way back to the stage of concentration factor design, based on maximizing this objective function.

The rapid convergence of $S_{N}^{\sigma}[f]$ to zero in smooth regions

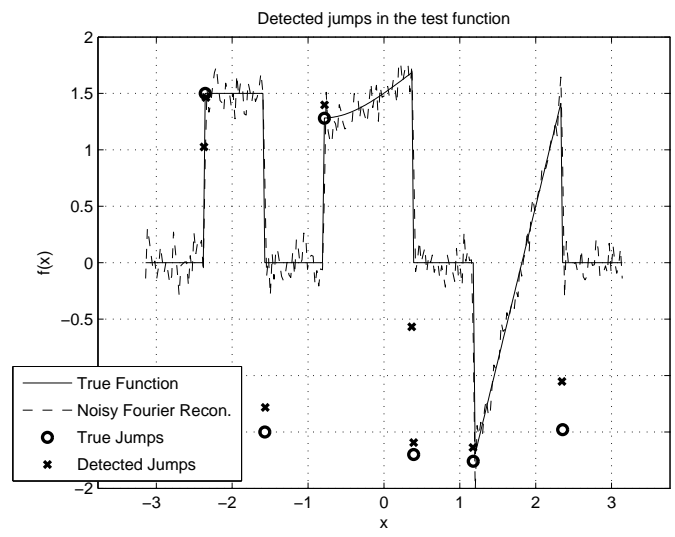

Fig. 5. Performance on a test function $\left(L=3, N=128, \rho^{2}=3.5\right)$

supports the use of the same $\mathcal{H}_{0}$ model in smooth segments of the signal. Near discontinuities, however, mitigation of "sidelobe effects" should be investigated to reduce clusters of false alarms near genuine jump points.

Little comparison of the concentration method to other nonparametric methods known in signal processing (e.g., digital spectral estimation for spectral line detection) has been undertaken. Casting this promising approach, which has already shown some effectiveness in MRI applications [6], in context of more well-established signal processing approaches is an appealing topic for further research.

\section{REFERENCES}

[1] A. Gelb and E. Tadmor, "Detection of edges in spectral data II - Nonlinear enhancement," SIAM Journal on Numerical Analysis, vol. 38, no. 4, pp. 1389-1408, Sep.-Oct. 2000.

[2] A. Gelb and E. Tadmor, "Detection of edges in spectral data," Applied and Computational Harmonic Analysis, vol. 7, pp. 101-135, 1999.

[3] A. Gelb and D. Cates, "Detection of edges in spectral data III - Refinement of the concentration method," Journal of Scientific Computing, vol. 36, no. 1, pp. 1-43, 2008.

[4] S. Engelberg and E. Tadmor, "Recovery of edges from spectral data with noise - a new perspective," SIAM Journal on Numerical Analysis, vol. 46, no. 5, pp. 2620-2635, 2008.

[5] S. Kay, Fundamentals of Statistical Signal Processing: Detection Theory, Prentice Hall, 1993.

[6] R. Archibald and A. Gelb, "A method to reduce the Gibbs ringing artifact in MRI scans while keeping tissue boundary integrity," IEEE Transactions on Medical Imaging, vol. 21, pp. 305-319, 2002. 